\title{
In Vivo Confocal Scanning Laser Microscopy
}

\author{
Oliver Stachs, Rudolf F. Guthoff, and Silke Aumann
}

\subsection{Introduction}

In vivo imaging of corneal cells has been pioneered by confocal scanning laser microscopy (cSLM) and the availability of commercial devices. With the combination of the Heidelberg Retina Tomograph (HRT, Heidelberg Engineering $\mathrm{GmbH}$ ) and the Rostock Cornea Module (RCM) a unique device using confocal point scanning technology was introduced in 2002 [1]. Since then, the HRT-RCM serves as a well-established instrument in experimental and clinical ophthalmology. The system represents an important device for ex and in vivo studies of animal and human corneas for a qualitative and partly quantitative analysis of corneal and limbal structures.

CSLM is a non-invasive imaging technique which generates transversal images (also called en-face images), with high resolution and excellent depth discrimination. Sequential acquisition of tomograms along the depth direction allows for $3 \mathrm{D}$ reconstruction of the volumetric data stack. The

O. Stachs · R. F. Guthoff

Department of Ophthalmology, Rostock University Medical Center, Rostock, Germany

S. Aumann $(\bowtie)$

Heidelberg Engineering GmbH,

Heidelberg, Germany imaging procedures cover a broad range of experimental and clinical applications. For example, cSLM serves as a multifunctional tool for corneal analysis of laboratory animals [2], it allows for assessing stromal changes in patients with keratoconus before and after cross-linking [3], for experimental full-thickness corneal 3D imaging [4], and for the quantification of morphology of epithelial cell layers and the subbasal nerve plexus (SNP) [5]. Intense research is focused on large-scale image reconstruction of the SNP [6-11], because it has the potential to serve as biomarker for early neurodegenerative changes [12].

While OCT imaging is very promising for corneal cross-sectional imaging, confocal scanning laser microscopy still offers superior lateral resolution combined with higher imaging quality. Various 3D reconstruction techniques of confocal image stacks are published. Volume imaging with a tandem scanning confocal light microscope is demonstrated by research as described in [13], but image quality and resolution are reduced compared to cSLM based methods. Volume imaging with the HRT-RCM is described in [5, $14,15]$.

This chapter summarizes the principles of confocal scanning laser microscopy and the technical implementation in the HRT-RCM. A selection of ophthalmological and non-ophthalmological applications is presented and current and future developments are shown. 


\subsection{Principle of Confocal Scanning Laser Microscopy}

The development of confocal imaging was largely driven by cell biology and the desire to observe biological events in vivo. In the early twentieth century, techniques for fluorescent staining of cellular structures have paved the way for fluorescent microscopy. With the intention to study neurons and their function at a cellular level, Marvin Minsky developed and patented the principle of confocal scanning microscopy in 1957 [16]. The fundamental idea is to illuminate the sample pointwise and to detect the reflected light pointwise, which means that the image build-up takes place sequentially. As a consequence, it was not before the 1980s that the full potential of this technique could be exploited: The rapid progress in laser and scanning technology as well as digital processing and storage abilities paved the way for a more efficient technical implementation of the confocal imaging principle. In vivo imaging at sub-cellular resolution and in real time became feasible.

In conventional microscopy, the specimen is flood-illuminated, meaning that backscattering occurs for the whole illuminated field within the tissue simultaneously. The optical imaging system generates an image where, for each tissue location, also stray light from the vicinity contributes to the final image. This crosstalk leads to a noisy background signal and impacts the visibility of small and faint tissue structures. For a thick specimen, many fluorophores-not only those within a thin slice in depth-contribute to the final image. Therefore, the preparation of very thin samples is needed to get any depth information at all.

Different to that, a confocal setup realizes point illumination and point detection, as is depicted in Fig. 12.1. The specimen is sequentially illuminated point by point either by using an illumination pinhole (also called aperture) or a focused beam. At each tissue location, light is reflected or backscattered and travels the same way back. It is separated from the incident beam by a beam splitter and the intensity is detected by a photo detector, which converts photons into a quantifiable electrical signal. Stray light is blocked by a pinhole in front of the detector. Therefore, only light from a small confined volume within the specimen-the confocal volume-can reach the detector. Out-of-focus light is strongly reduced, improving image resolution and contrast considerably. The detected signal is confined to a very limited depth range within even a thick specimen. Therefore, confocal imaging is commonly referred to as optical slicing or optical sectioning, which underlines its similarity to histology while emphasizing its non-invasiveness. The term confocal refers to the fact, that the planes of illumination and detection are optically conjugated. Both are in focus simultaneously.

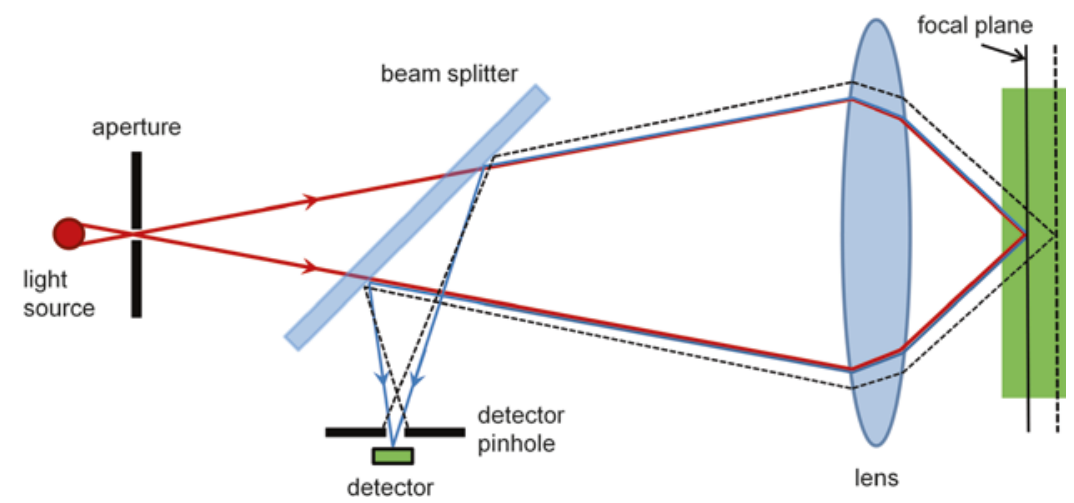

Fig. 12.1 The principle of confocal illumination and detection: Using an aperture, a light source is imaged to illuminate a single spot within the specimen. With the same objective lens the backscattered (or fluorescent) light originating from this spot is imaged on the detector. The detector pinhole removes all light from outside the focal plane 
Confocal reflection imaging reveals the backscattering or reflecting properties of the specimen. Elastic scattering preserves the photon energy, i.e. the wavelength of the backscattered light is not shifted with respect to that of the illuminating light. However, the backscattering efficiency or scattering cross section generally is wavelength dependent. In confocal fluorescence imaging the tissue is either stained with fluorophores or shows intrinsic (auto-) fluorescence. Additional optical barrier filters are needed to block the excitation light, thereby allowing for selectively detecting fluorescence light of a specific wavelength or wavelength range. Some selected applications of confocal fluorescence imaging in ophthalmology can be found in Chap. 2.

In vivo imaging of the eye is particularly demanding due to eye motion, which makes acquisition speed crucial. The first confocal scanning laser ophthalmoscope was presented by Webb et al. [17] in the 1980s, demonstrating imaging of the ocular fundus. Since then, retinal confocal imaging has developed into a routine application in clinical practice. Without any additional measures (i.e. adaptive optics), the optical resolution of retinal images is limited to about $15 \mu \mathrm{m}$. Both the finite pupil size and optical aberrations of the eye account for this and imaging at sub-cellular level cannot be achieved.

In contrast, confocal microscopy is feasible for the anterior segment of the eye. As the eye is not part of the imaging system - or only to a very limited extent-microscope lenses with high numerical aperture (NA) can be used. Employing a tandem scanning confocal microscope, Cavanagh et al. demonstrated in vivo imaging of the human cornea in 1989 [18]. Confocal imaging provides a much higher optical resolution and optical sectioning capability than a slit-lamp biomicroscope, which is limited to a magnification of about $40 \times$ and an optical resolution of about $20 \mu \mathrm{m}$.

Different types of confocal microscopes have been described for use in ophthalmology, which mainly differ in their principle idea to scan the specimen. The tandem scanning confocal microscope (TSCM) uses the basic idea of the Nipkow disc - a rotating disc equipped with a spiral array of pinholes and was originally developed by Petran and Hadravsky [19]. The specimen is illuminated and sampled in parallel by a set of conjugate pinholes arranged along concentric circular traces. A bright light source is required, usually a xenon or mercury arc lamp. This allows for true-color and real-time imaging, but has the disadvantage of a very low light throughput and therefore rather low image quality and contrast. The image is observed with the eye. The system is no longer commercially available.

The concept of the scanning-slit confocal microscope (SSCM) was developed by Thaer et al. [20, 21]. A sheet of light is scanned over the back focal plane of the microscope objective, thereby illuminating the specimen with a slit of light and detecting the backscattered light with a line sensor. The parallel illumination and detection allows for considerably increasing acquisition speed but with the drawback of reduced optical resolution. Confocality is maintained only along one spatial direction, resulting in anisotropic lateral resolution and degraded depth resolution.

Nowadays, laser diodes are commercially available at a variety of different wavelengths from the visible to the infrared region. Due to their compact design and simple control they can easily be integrated into imaging systems. When combined with beam shaping optics, laser can provide small spot sizes and high radiant flux, thereby allowing for a very efficient illumination and detection. Spot illumination eliminates the need for an illumination pinhole. Such confocal point scanning systems have superior signal-to-noise characteristics and allow for a laterally isotropic resolution and for an optimum depth sectioning ability. Nevertheless, in order to reach high frame rates the requirements for the scanning unit are demanding. The HRTRCM represents a confocal microscope of this type and will be introduced in more detail in the next section.

The theory of confocal microscopy was more formally developed and extended by Wilson and Sheppard [22]. The superior optical performance of a confocal setup is reflected by its point-spread 
function (PSF). Generally, the 3D PSF describes the intensity distribution in the image space of an optical imaging system resulting from a point source object. Depending on the imaging properties, the point image may be blurred in the presence of optical aberrations, in the ideal case it is limited by diffraction only. In this case, the central maximum of the 3D PSF, which contains nearly $90 \%$ of the total energy available, can be described as an ellipsoid of rotation.

For considerations of resolution and optical slice thickness, it is useful to define the area in which the intensity of the PSF in lateral and axial direction has dropped to half of its central maximum, respectively. In the transversal plane, this condition confines an area corresponding to the central bright disc of the concentric diffraction pattern. In the perpendicular plane it defines a double cone or hourglass along the optical axis, i.e. in depth z. The two characteristic measures are given by the beam waist $\Delta x$ and the confocal parameter $b$, which corresponds to half the Rayleigh range $z_{R}$.

As is depicted in Fig. 12.2, the PSF and thereby the resolution depend on the wavelength and the numerical aperture (NA) of the microscope lens or any other focusing optics. The numerical aperture is a measure to describe the acceptance cone or light-gathering ability of an objective lens. The shorter the focal length of the objective lens and the larger the beam diameter

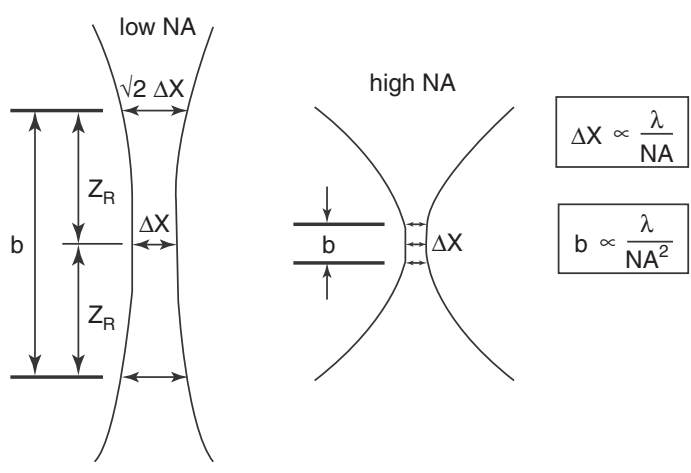

Fig. 12.2 The numerical aperture (NA) determines the lateral image resolution and the depth of field. Lateral resolution is characterized by the beam waist $\Delta x$ of the focused laser spot, depth of field is specified by the confocal range $b$ or Rayleigh range $z_{R}$ at the entrance pupil of the lens, the higher is the NA. In addition, the acceptance cone can be increased by using immersion objectives, the NA being proportional to the refractive index $n$ of the immersion medium.

In contrast to conventional microscopy, the optical performance of the confocal system is determined by two PSFs: the illumination $P S F_{\text {ill }}$, which describes the intensity distribution of the scanning laser focus in the object space and the detection $P S F_{d e c}$, which accounts for the projection of a point object into the image space. Both depend on the wavelength and numerical aperture (NA) as was shown above. In addition, $P S F_{d e c}$ is determined by the size of the detection pinhole. The imaging properties of a confocal system are defined by the final $P S F_{t o t}$, which mathematically is described as the product of both single PSFs. It can be shown that $P S F_{\text {tot }}$ is always less than or equal to $P S F_{i l l}$, whereby the size of the detection pinhole plays the decisive role in resolution and depth discrimination. It controls the continuous transition from object information suppressed and such made visible. If the pinhole size is larger than the central disc of the diffraction pattern described above, the resolution is governed by $P S F_{i l l}$ and there is no difference compared to conventional imaging. As the pinhole becomes smaller, $P S F_{d e c}$ approaches $P S F_{\text {ill }}$, which results in a superior resolution compared to conventional imaging. It can be shown, that in the limit of $P S F_{d e c}=P S F_{i l l}$, the lateral resolution and the depth discrimination are both improved by a factor of about 1.4 [23].

In practice, there is a trade-off between resolution and detection sensitivity. The size of the pinhole is adapted as to narrow the $P S F_{d e c}$ as much as possible while still allowing enough photons passing through and being detected. The main benefit of confocal imaging is not the rather modest increase in optical resolution. The fundamental improvement results from the suppression of out-of-focus light, which allows to optically slice a specimen. Then, a depth (z) position can be assigned to each image and volumetric imaging for intact and living specimens becomes feasible. 


\subsection{In Vivo cSLM with the Rostock Cornea Module}

The Heidelberg Retina Tomograph (HRT, Heidelberg Engineering $\mathrm{GmbH}$ ) was the first commercial confocal scanning laser ophthalmoscope (cSLO) which was widely used in a clinical setting for glaucoma diagnosis. It was brought to market in the early 1990, the next generations of the device, called HRTII and HRT3, were released in 1998 and 2005, respectively.

The HRT uses point scanning to acquire a 3D stack of tomograms of the optic nerve head, which allows for its topographic analysis. A normative database is used to compare individual data with those of healthy and early-glaucoma patients. Follow-up examinations and progression analysis have shown to be highly beneficial in glaucoma care. The device enabled for routine screening in a clinical and medical practice setting.

In the early 2000, Stave et al. from the Rostock Eye Clinic presented an optical attachment to the HRTII [1], by this way allowing for microscopic imaging of the cornea. Based on their experience, Heidelberg Engineering developed the Rostock Cornea Module (RCM) which entered the market in 2004. Designed as an accessory for the HRTII, it allows for using the same imaging platform for different ophthalmological applications. The RCM has also been released for the HRT3. As presented in Fig. 12.3, the RCM is attached to the objective of the cSLO.

The HRT camera head integrates all core components required for confocal imaging-a light source, a scanning unit and a photodetector. Although the principal setup is the same for corneal imaging, the RCM objective changes the imaging parameters in a fundamental way. Image generation is briefly explained in the following section, where differences between corneal and retinal imaging are pointed out.

The schematic optical setup of the HRT-RCM is presented in Fig. 12.4. Essentially, the HRT is changed from a confocal scanning laser ophthalmoscope (cSLO) to a confocal scanning laser microscope (cSLM) by placing the RCM objec-

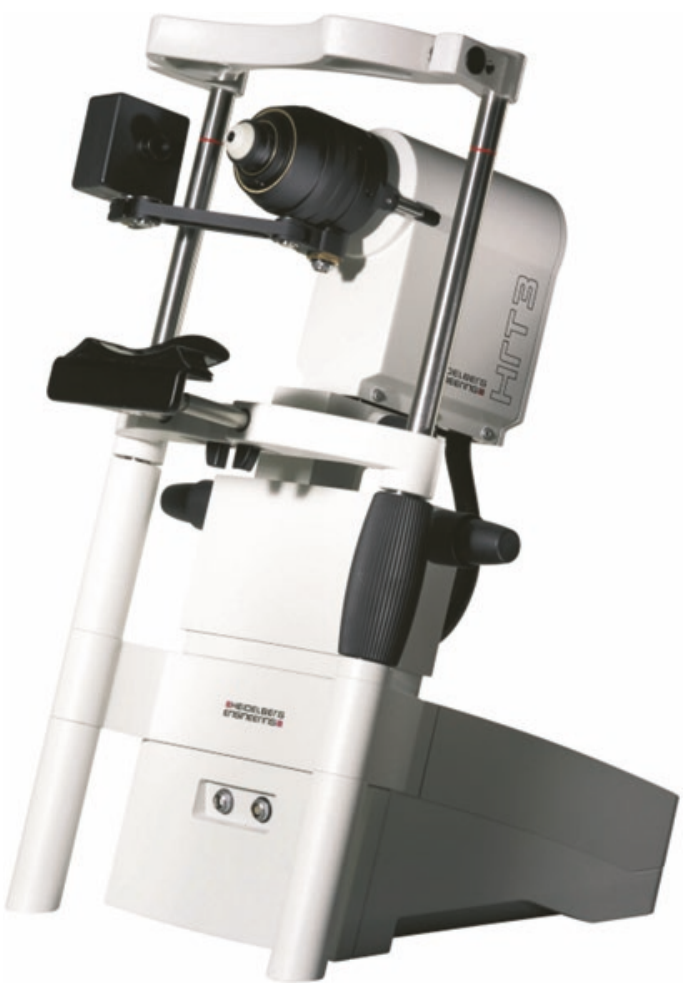

Fig. 12.3 The Heidelberg Retina Tomograph (HRT3) is a confocal scanning laser ophthalmoscope which allows for analysis of the optical nerve head. In combination with the Rostock Cornea Module (RCM), which is attached to the HRT, confocal microscopy of the cornea is feasible

tive in the entrance pupil of the HRT. In retinal imaging, the pupil of the examined eye is positioned at this pupil location.

The scanning and detection scheme are the same for both, the HRT and the HRT-RCM. The collimated beam of a red laser diode $(670 \mathrm{~nm})$ is deflected by a beam splitter and enters the $x-y$ scanning unit. To acquire a two-dimensional transversal image of the specimen the beam is deflected in two perpendicular directions. A resonant x-scanner supports a line rate of $16 \mathrm{kHz}$, a galvanometer scanner is used for the slower $y$-scan, thereby providing a raster scan.

The scan pupil is relayed by means of telescope optics to the entrance pupil of the RCM objective. The core component of the RCM is a water-immersion microscope lens with a high numerical aperture. It provides a focus at a distance of a few millimeters in front of the apex 


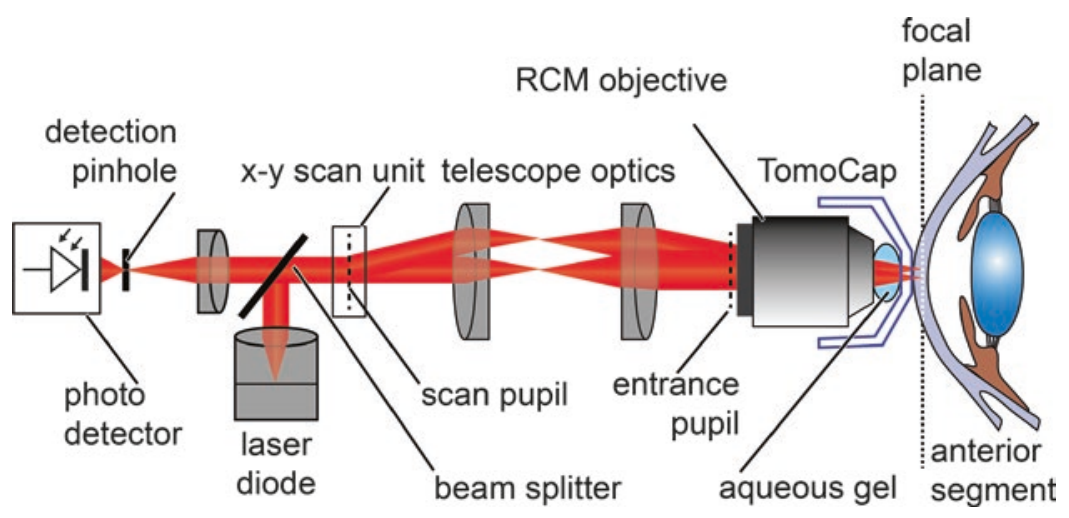

Fig. 12.4 Principle setup of the confocal scanning laser microscope (cSLM): The HRT-RCM uses the light source and the scanning and detection units of the HRT. The

of the lens. The cornea or any other tissue under examination is positioned in the focal plane. From each illuminated point, backscattered light travels the same path back and is separated from the illumination path by the beam splitter. The signal is detected by an avalanche photo diode, which has a detection pinhole to ensure confocality. The output signal of the photodetector is sampled with a pixel clock of $12 \mathrm{MHz}$ while the $\mathrm{x}-\mathrm{y}$ scanning unit performs a transversal raster scan. Each digitized value is assigned to the corresponding pixel in the confocal image. The final image consists of $384 \times 384$ pixels and represents the backscattering properties of a thin transversal slice within the tissue. A frame rate of about $30 \mathrm{~Hz}$ allows for real-time imaging.

For volumetric imaging the focal plane has to be shifted axially to change the depth position of the image. The RCM has a mechanical z-feed, which allows for manual fine adjustment of the focus plane within a range of approximately $1.7 \mathrm{~mm}$. In addition, the focal plane can be shifted optically within a range of $80 \mu \mathrm{m}$.

In the following, some important imaging details and components are described in more detail. The interesting reader can find more details on the HRT in Chap. 2. As can be recognized from Fig. 12.4, the pivot point of the scanning unit is optically transferred to the entrance pupil of the RCM objective. This pupil can be seen as the origin of a collimated beam being deflected by virtual scanners. Ideally, it is brought to coin-
RCM objective changes the imaging parameters to allow for microscopic imaging. The water immersion lens is coupled to the cornea with a single-use contact element

cide with the entrance pupil of the microscope lens. The large numerical aperture of the microscope lens (Achroplan 63×/W, NA 0.95, Zeiss) provides a high optical resolution and depth discrimination. The axial position of the focal plane measured from the front lens' apex equals $2.2 \mathrm{~mm}$ and is called working distance.

The cornea or specimen is optically coupled to the imaging device using an aqueous gel of high viscosity and a sterile, single-use contact element-the TomoCap. The TomoCap is a thin, transparent cap made of PMMA, which touches the cornea with its flat front surface during the imaging procedure.

In contrast to retinal imaging, where the eye is an integral part of the imaging process, cSLM allows to set the magnification and resolution independent of the imaged object. Essentially, the microscope lens determines the optical parameters and must be selected accordingly.

The cornea has an average refractive index of $\mathrm{n}=1.376$, the main constituent being water. Therefore, water-immersion microscope lenses are the best choice. They are optimized for imaging in aqueous solution, i.e. for a medium with a refractive index of 1.33 . They minimize spherical aberration while imaging through an aqueous specimen and they potentially provide higher numerical apertures compared to non-immersion types, as the focus is sharper and the acceptance angle to collect backscattered light is larger (by a factor of 1.33). 
Any change in refractive index along the optical pathway gives rise to back reflection, which introduces signal loss and potentially can lead to ghost reflections. Therefore, the aqueous gel also has to be applied to bridge the air gap between the microscope's apex and the TomoCap.

In the following, the standard workflow of a cornea exam and the main software functionality are described briefly. It is common practice to use topical anesthetic during the imaging procedure to minimize discomfort of the patient. In addition, a drop of eye gel is given into the patient's eye. After the instrument has been prepared the patient is positioned in the headrest. The RCM objective is carefully adjusted to approach the patient's cornea. This procedure is monitored by a small external camera as is depicted in Fig. 12.5 (right). As soon as the TomoCap touches the cornea, cell structures become visible (see Fig. 12.5, left). With the manual z-feed, the focal plane can be shifted through the whole cornea, thereby sequentially imaging single corneal cell layers.

The contact method provides a way to reduce eye motion and enables to assign depth values to the cSLM images due to axial stabilization. The actual focus position is measured with respect to a reference plane, which enables to detect certain cell layers in a more systematic way.
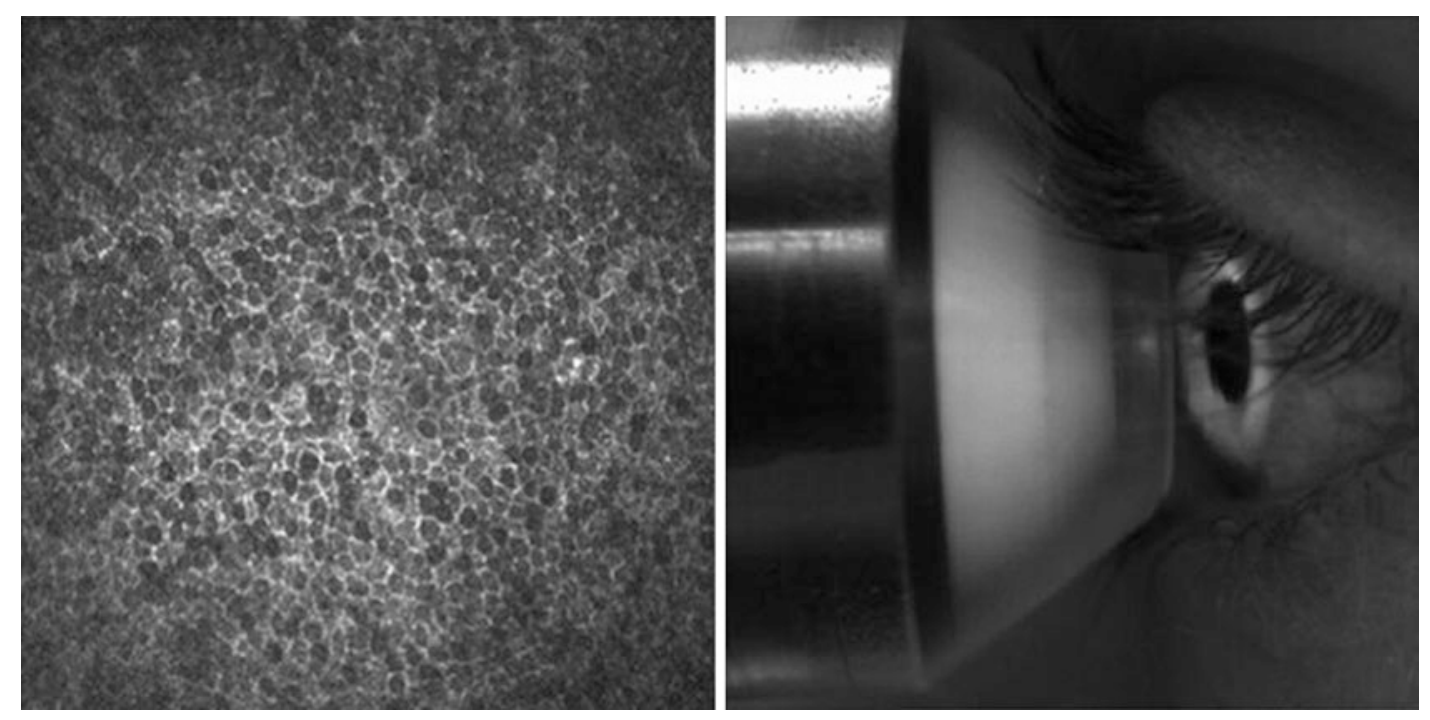

The HRT-RCM supports the acquisition of single images, movies and volume scans. A volume scan (also referred to as Z-series) is a sequence of optical sections at an equidistant spacing of about $2 \mu \mathrm{m}$. Forty images are collected while the focus of the HRT incrementally changes by means of a stepping motor. This automated volume scan is limited to a depth range of about $80 \mu \mathrm{m}$.

The HRT-RCM features a field of view of $400 \mu \mathrm{m} \times 400 \mu \mathrm{m}$, a lateral resolution of $1-2 \mu \mathrm{m}$ and an axial resolution of about $4 \mu \mathrm{m}$. It thus enables imaging on the subcellular level with almost isotropic resolution. Compared to retinal cSLO imaging the lateral resolution is increased by about the tenfold, the depth of field is decreased by about one hundred. Very sharp and narrow slicing of the specimen is feasible, which is essential to access single epithelial cell layers of the cornea. As was already pointed out, the large numerical aperture (NA) of the microscope lens accounts for the high resolution, whereas in retinal imaging it is limited by the rather low NA of the eye and the diameter of the imaging beam. This principle difference was schematically presented in Fig. 12.2.

Cellular imaging requires a microscope lens with a short focal length, which also limits the working distance (WD). The depth of the layer

Fig. 12.5 The user interface of the HRT-RCM software displays two images simultaneously: the cSLM image (left) and the monitoring image of the external camera (right) 
which can be accessed within the tissue is confined to that distance. A WD of $2.2 \mathrm{~mm}$ is sufficient to image cornea, limbus or sclera. But deeper tissue of the anterior segment, e.g. the lens epithelium, can only be imaged with a microscope lens of lower NA, which then reduces resolution.

The lateral field of view (FOV) is determined by the maximum scan angle and the focal length of the microscope lens, which is proportional to the FOV. Therefore, subcellular resolution is feasible only for small FOVs in the order of several hundreds of micrometers. Field of view and resolution cannot be optimized independently.

However, it is possible to stitch single frames to larger field of views. The mosaicking feature of the HRT-RCM software enables the acquisition of realtime composite images. These comprise a maximum area of 64 single frames resulting in a total FOV of about $3 \mathrm{~mm} \times 3 \mathrm{~mm}$. Each single frame is inspected for features that match the composite and is then aligned and added to the composite. In general, random and involuntary eye movement is not sufficient to fill this large frame. The patient needs eye guidance e.g. by a moving fixation target. Composite images have been shown for the subbasal nerve plexus, see the following section.

The cornea is a highly transparent tissue, which backscatters only about $1 \%$ of the incident light in the visible range [24]. Its main constituent is water which practically shows no absorption in the visible spectrum. The main contribution to light attenuation while passing through the cornea comes from scattering rather absorption.

In the field of biomedical optics Rayleigh scattering and Mie scattering are commonly used to describe light-tissue interaction.

Rayleigh scattering is referred to as scattering by small particles that have a refractive index different from the surrounding medium and a particle size much smaller than the wavelength. Also, spatial fluctuations in density on this scale may cause continuous variations in refractive index. Rayleigh scattering shows equal intensities for forward and backward scattered light, which are inversely proportional to the fourth power of wavelength.

Mie scattering is commonly referred to as scattering by particles comparable in size to the wavelength. It shows a weaker dependence on wavelength and preferably takes place in the forward direction.

Most biological tissues neither show pure Rayleigh nor Mie scattering: the photons are preferably scattered in the forward direction, i.e. scattering is highly anisotropic [25]. On the other hand, the observed wavelength-dependence is generally stronger than predicted by Mie theory [26]. Therefore, mathematical modeling of laser interaction with tissue or Monte Carlo simulation may be useful to deduce optical scattering properties. Structural modifications in tissue can manifest themselves in changes in confocal signal intensity and it is a major field of research to link macroscopic optical properties with microscopic tissue architecture.

The transparency of the cornea is attributed to an extremely regular structure of collagen fibrils of homogenous diameter [27]. The cornea is about $500 \mu \mathrm{m}$ thick, of which about $90 \%$ consists of collagen fibers, interstitial substance and keratocytes.

Corneal epithelium is comprised of superficial cells, wing cells and basal cells. The epithelial cells have bright cell boundaries and the size varies from about $40 \mu \mathrm{m}$ for the superficial cells to $8-10 \mu \mathrm{m}$ in the basal epithelium.

As is depicted in Fig. 12.6 (a) the superficial epithelial cells have a polygonal shape with a bright nucleus and bright cell borders. The wing cells $(b, c)$ appear with bright cell borders and dark cytoplasm. The nucleus can be distinguished only with difficulty. There is minimal variation in shape and size. The cell borders of the basal cells (d) are very bright, the nucleus is not visible. The reflectivity of the cytoplasm is rather inhomogeneous.

Immediately posterior to the basal epithelium an amorphous membrane is located, called Bowman's layer (e). Bowman's layer is made of collagen fibers and contains unmyelinated c-nerve fibers. It is about $10 \mu \mathrm{m}$ thick and the confocal images appear featureless and grey, with discrete beaded nerve bundles running parallel to the corneal surface.

The keratocyte nuclei of the stroma (f, g) are 5-30 $\mu \mathrm{m}$ in diameter and are hyperreflective. In normal tissue, the collagen fibers and the inter- 

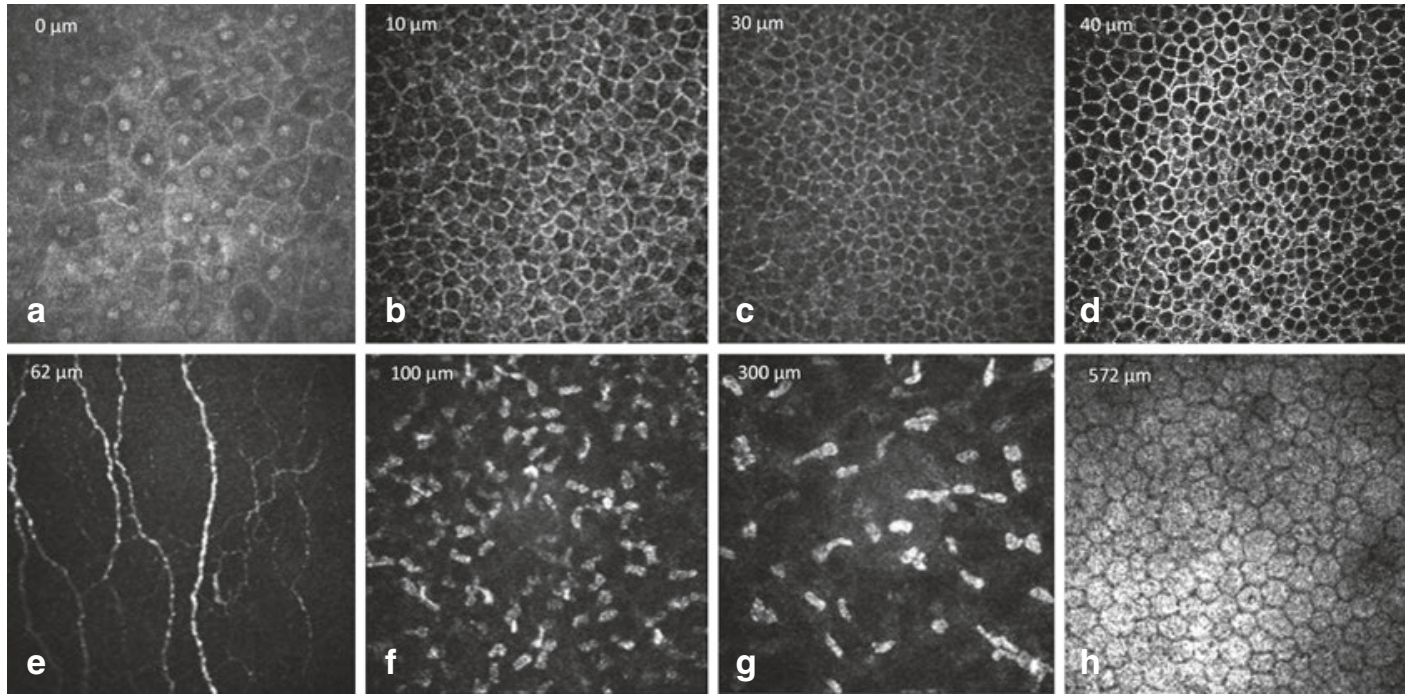

Fig. 12.6 Normal findings in ocular surface tissues: (a) superficial cells, (b) upper wing cells, (c) lower wing cells, (d) basal cells, (e) subepithelial nerve plexus, (f) anterior stroma, (g) posterior stroma and (h) endothelium

stitium appear as gray amorphous background. In the anterior stroma myelinated nerve fibers can be occasionally visualized at lower density and higher thickness. Between the posterior stroma and the endothelium Descemet's membrane appears as an acellular layer. It has a hazy appearance and becomes more visible with increasing age.

The cornea endothelium (h) is a single layer of hexagonal or polygonal shaped cells, about 4-6 $\mu \mathrm{m}$ thick and $20 \mu \mathrm{m}$ in diameter, appearing with bright cell bodies and hypo-reflective cell boundaries. The cell nuclei are rather difficult to recognize. With increasing age endothelial cell density reduces and polymegathism increases.

\subsection{Ophthalmological Applications}

For conventional microscopic evaluation of biological tissues usually thin slices with $2-5 \mu \mathrm{m}$ thickness were cut, stained with various chemicals (dyes) and examined by light transmission microscopic techniques at high magnification (400-1000-fold).

For in vivo examination of semitransparent tissues slit lamp biomicroscopy is the standard technique since over a century. The inventor Allvar Gullstrand a Norwegian physicist and ophthalmologist was awarded the Noble price mainly for this breakthrough invention 1911. In this technique, optically cut planes are orientated sagittally and observed by a binocular microscope with the magnification up to 50-fold. With this magnification single cell resolution is impossible. Nevertheless, the majority of corneal diseases could be diagnosed that way and treatment follow up was easily possible in most of the patients.

The confocal in vivo microscopy images deal with the same unprocessed biological structures but

(1) with a magnification of approximately 800fold which clearly allows single cell evaluation and

(2) the optical section directed parallel to the corneal surface which means perpendicular to the slit lamp image.

Both phenomena made it necessary to create new standards for the interpretation of images obtained by in vivo confocal microscopy of the cornea. In the meantime, this is documented in various textbook chapters and atlases, e.g. [28]. 
In general, there are a number of very interesting applications for corneal diagnostics covering a broad range of clinical applications:

- Corneal nerve degeneration and regeneration

- Chirurgical interventions

- Corneal grafting

- Refractive surgery

- Contact lenses

- Diabetes mellitus

- Keratoconus

- Ocular surface disease

In the following, three examples will be given to underline the clinical benefit of in vivo corneal confocal microscopy. Then, short overviews of cSLM in animal and interdisciplinary research are given respectively.
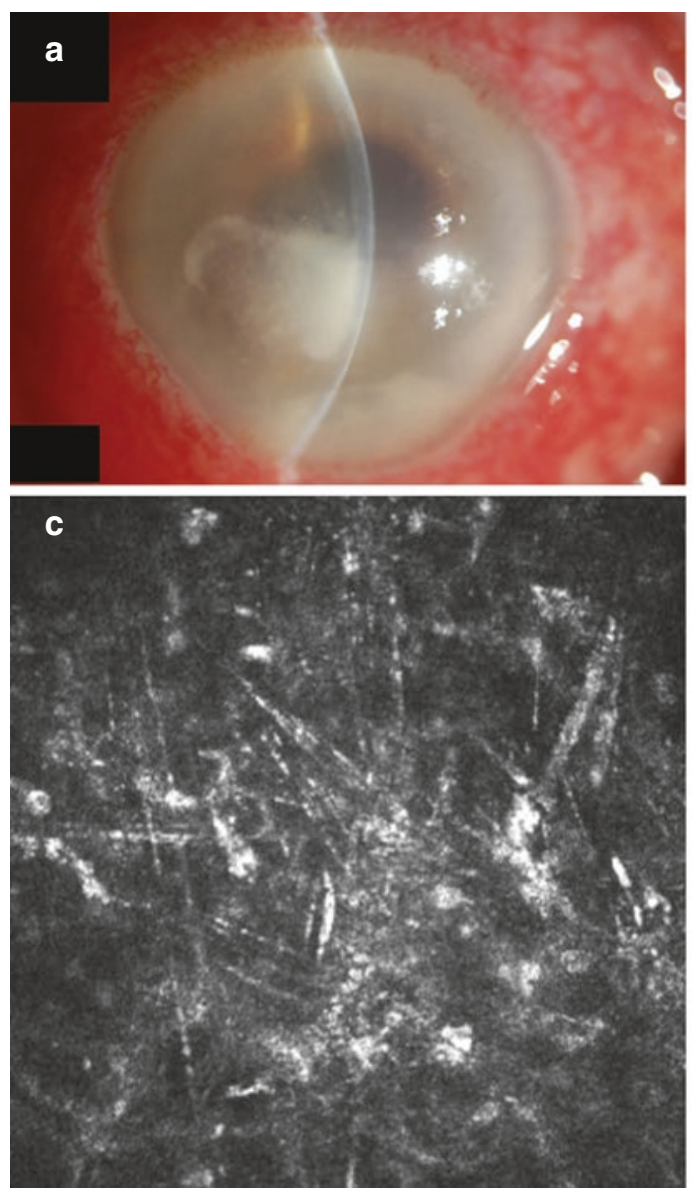

Fig. 12.7 Slit lamp photograph of corneal ulcers of unknown aetiology $(\mathbf{a}, \mathbf{b})$ and confocal images of these lesions showing spindle shaped activated keratocytes, (c)

\subsubsection{Diagnoses of Keratomycosis}

Fungal keratitis plays a major role in many developing countries, where early diagnoses and treatment are often not easily available. Even in relatively infrequent infections in industrialized countries proper management of fungal keratitis due to prolonged diagnostic procedures ends up with devastating results [29].

In vivo confocal microscopy has proven to be an excellent way for instant diagnoses avoiding long lasting preparations of sample cultures. CSLM supports the differential diagnosis of non-fungal keratitis with activation of keratocytes and instantly proven fungi in a corneal ulcer, as is demonstrated in Fig. 12.7.
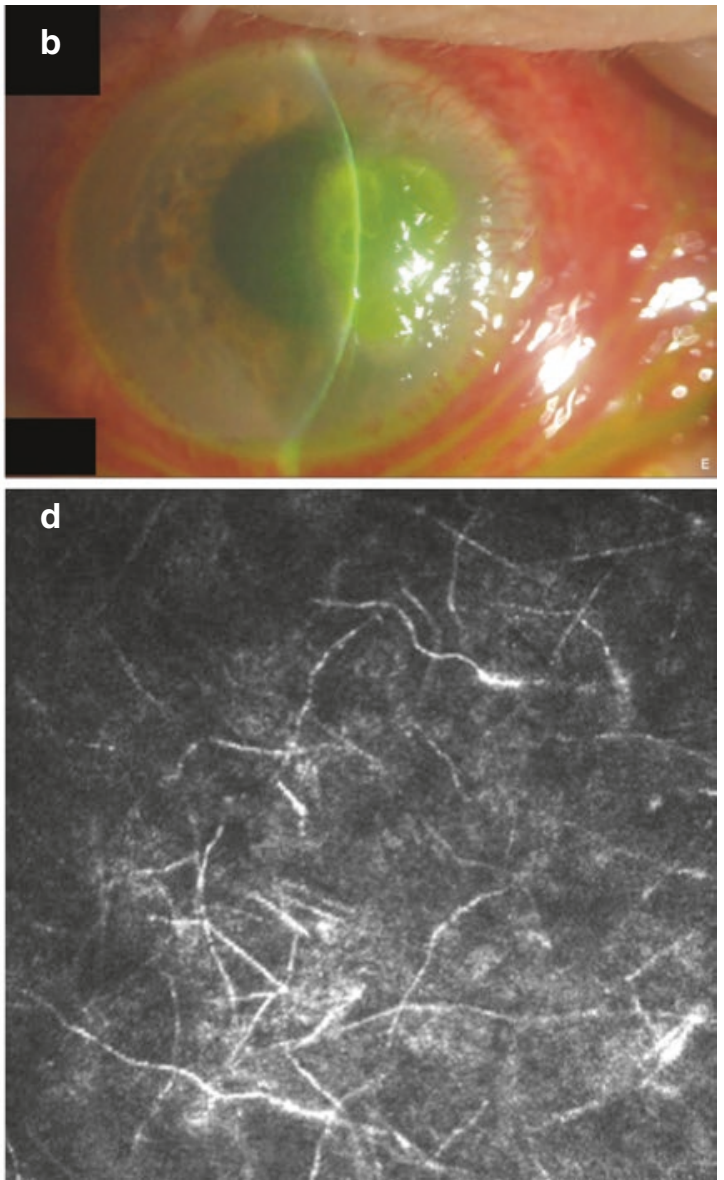

corresponding to (a) and typical fungal elements (d) corresponding to (b) 


\subsubsection{Subbasal Nerve Plexus}

The ophthalmological evaluation of the subbasal nerve plexus of the cornea might add value to the diagnosis of a variety of diseases.

Corneal nerves are affected in cases of

- limbal stem cell deficiency

- infection

- corneal surgery

- keratoconus

- diabetes

- lysosomal storage diseases

- keratitis.

Especially, small fiber neuropathy in diabetic patients is a main cause for limb amputation both in the industrialized and in the developing countries [30]. Clinical quantification of the disease with widely accepted methods like neuropathy symptoms score (NSS) and neuropathy deficit score (NDS) as well as skin sensitivity measurements with a so-called monofilament delivers positive results only in patients where a considerable nerve fiber damage has already taken place $[12,31]$.
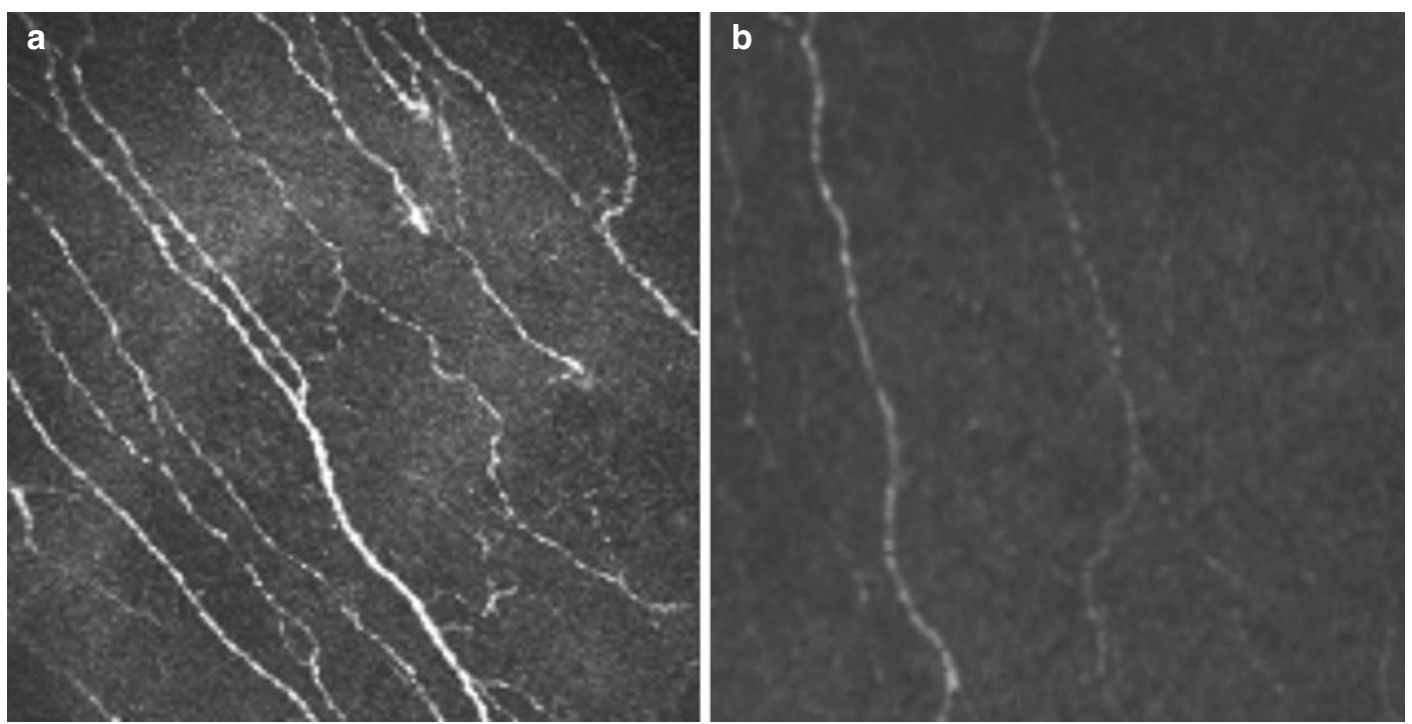

In vivo confocal microscopy provides optical slicing parallel to the surface of the cornea. This offers an ideal physical prerequisite to display and to quantify structures of the subbasal nerve plexus in a well-defined anatomical plan. The nerve plexus is located between Bowman membrane and the basal lamina of the corneal epithelial cells (see Sect. 12.6). Numerous publications deal with this structure as a very early surrogate marker for diabetic neuropathy [12]. Examples of normal and rarefied nerve fibers in this layer are given in Fig. 12.8.

Imaging of the subbasal nerve plexus might pave the way for new treatment strategies and more effective prevention of this serious disease. Diabetes-induced complications could thereby reduced.

\subsubsection{Corneal Keratocyte: A Neglected Entity of Cells}

Keratocytes are specialized fibroblasts representing about $10 \%$ of the volume of the corneal stroma. Their cytoplasm with a total diameter of up to $100 \mu \mathrm{m}$ is highly transparent whereas

Fig. 12.8 Single image from the subbasal nerve plexus of the cornea in an individual without diabetes (a) and in an individual showing a decrease in the nerve density and nerve length (b) 
nuclei with a diameter of about approximately $10 \mu \mathrm{m}$ are the main light scattering elements of the cornea.

Vogt described them as "corpusculi cornea" in his fundamental textbook on slit lamp biomicroscopy in 1930. Keratocytes are placed in a network of highly organized and different shaded collagen lamellas which are also invisible even for confocal microscopy due to their complex 3D arrangement avoiding any light scattering or reflectance.

In vivo confocal microscopy easily displays keratocyte nuclei. The following mean cell densities were found: in the basal epithelium $6000 \pm 1080$ cells $/ \mathrm{mm}^{2}$, anterior stroma $765 \pm 262$ cells $/ \mathrm{mm}^{2}$, mid stroma $347 \pm 64.4$ cells/ $\mathrm{mm}^{2}$, posterior stroma $315 \pm 57.2$ cells $/ \mathrm{mm}^{2}$, and endothelium $2720 \pm 367$ cells $/ \mathrm{mm}^{2}$ [32]
There is still no theory why the density of keratocytes varies according to their localization and whether there are clear keratocyte subtypes in different layers of the cornea [33].

Various noxes such as local mechanical ones by epithelial erosions or toxic ones from free oxygen radicals in corneal $\mathrm{x}$-linking change the optical properties of the cytoplasm of keratocytes dramatically, as is depicted in Fig. 12.9. There is evidence that these morphological changes appear in the process of cell death when apoptotic vesicles in the keratocyte stroma turn transparent cellular part into accumulation of highly scattering elements [34]. These ghost cells finally disappear totally and after days are replaced by spindle shaped migrating keratocyte subtypes migrating from adjacent corneal areas.
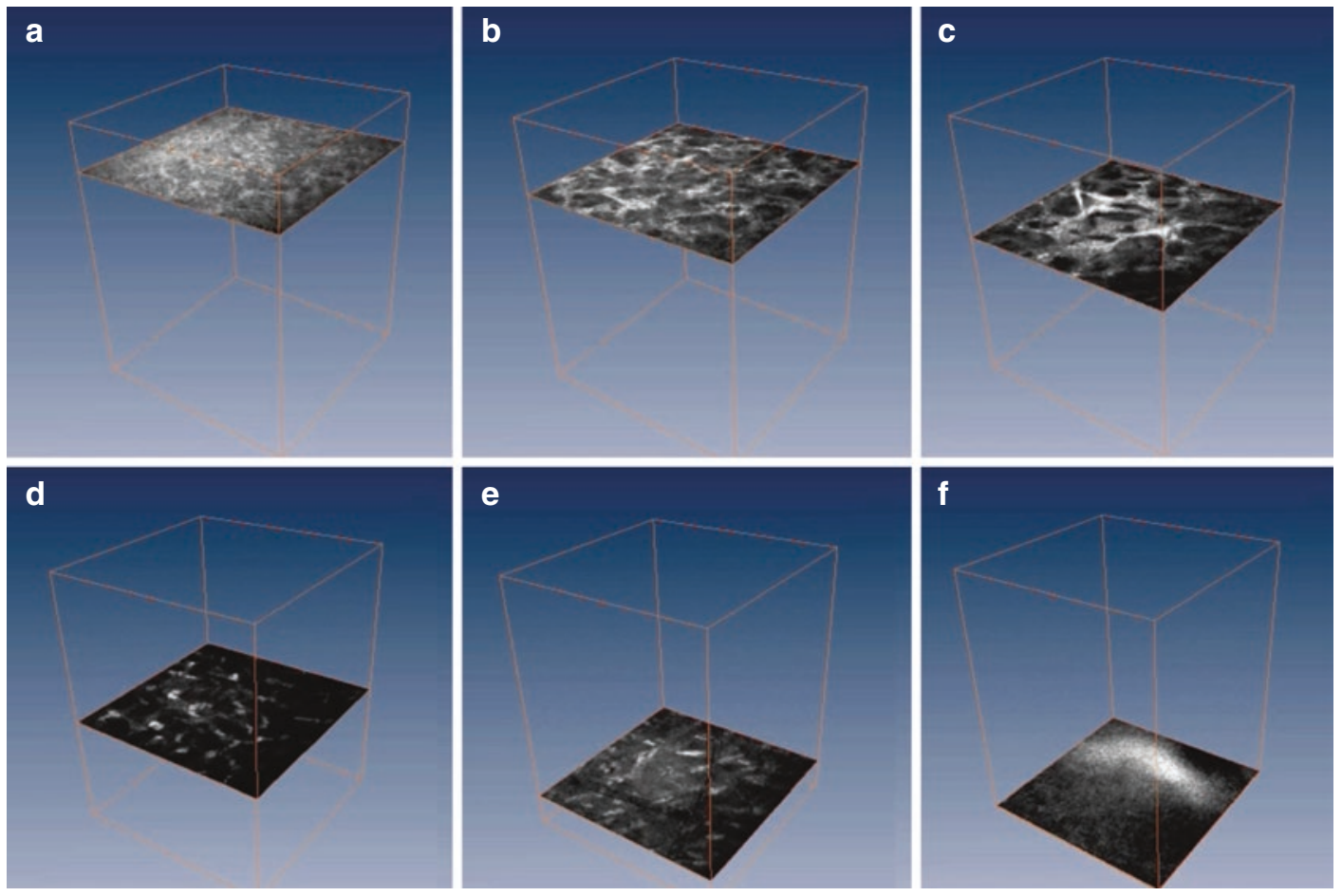

Fig. 12.9 Volumetric cSLM representation in an individual after $\mathrm{x}$-linking showing alterations in the anterior part of the cornea $(\mathbf{a}-\mathbf{c})$ and a normal appearance in the posterior part $(\mathbf{d}-\mathbf{f})$ 


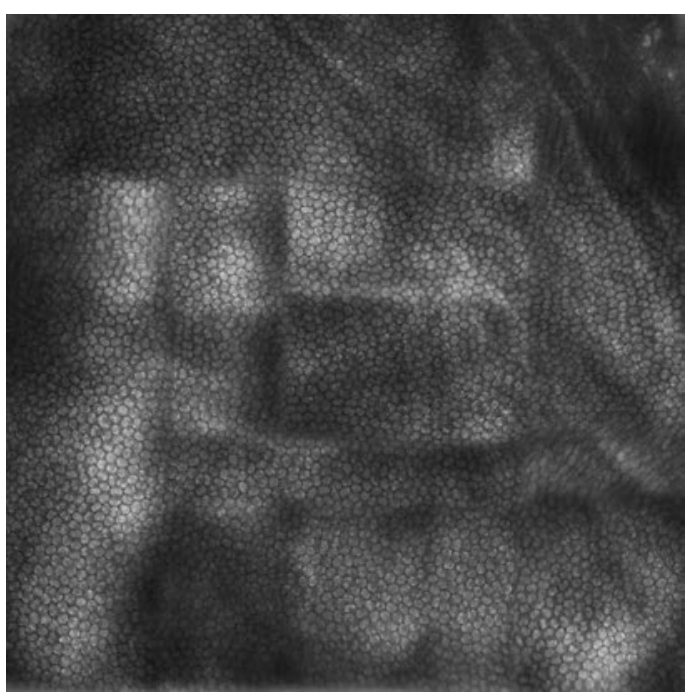

Fig. 12.10 Mosaicking cSLM in animal research showing the rabbit endothelium $(2.5 \mathrm{~mm} \times 2.5 \mathrm{~mm})$

\subsection{4 cSLM for Animal Studies}

In vivo cSLM can be used for a wide range of applications in animal studies and veterinary research, see Fig. 12.10. Data are available on the normal corneal anatomy of rabbits [35], in rats [36, 37] and mice [38, 39]. Interspecies comparisons were published by Labbe and Reichard comparing the anatomy of laboratory animals (rabbits, rats, mouse) [2, 40].

There are a large number of publications covering the full range of corneal research. In vivo cSLM was used for animal studies to assess the corneal surface after the application of topical drugs or preservatives [41] to analyze corneal response after refractive surgery [42, 43] and alterations after contact lens wearing [44, 45]. Furthermore, a number of studies deal with mice models to investigate diabetes-induced small fiber neuropathy [46, 47]. Hovakimyan et al. have investigated matrix-based regenerating agent for corneal wound healing after collagen cross-linking [34].

\subsubsection{Interdisciplinary Research}

The ability of cSLM to generate high-resolution in vivo images of the highly innervated cornea has drawn pronounced interest as it allows for using this innovative technology as a biomarker for disease staging in humans.

For instance, cSLM has visualized and highlighted significant morphological alterations of the subbasal nerve plexus in diverse diseases such as Parkinson's disease and progressive supranuclear palsy [48], amyotrophic lateral sclerosis, chronic migraine [49], multiple sclerosis [50, 51] and amyloid neuropathy [52]. But diabetic peripheral neuropathy, in particular, has been the main focus of various studies [12] because characteristic morphological alterations of the subbasal nerve plexus already occur at an early stage of diabetic peripheral neuropathy [53, 54]. In conclusion, cSLM has the potential to reveal reliably the biomarkers for the early assessment of diabetic peripheral neuropathy (DPN).

An own pilot study has confirmed that changes of the corneal subbasal nerve plexus can be seen as a predictor for diabetic Charcot foot deformity, see Fig. 12.11. The combined clinical assessment in multiple myeloma of peripheral neuropathy in parallel with the investigation of morphological nerve fiber changes using in vivo cSLM is a new approach allowing highly sophisticated detection of morphologic neuronal changes.

In summary, there is a high evidence of cSLM in general clinical practice as a noninvasive method of assessing peripheral neuropathies, monitoring inflammatory states and clinical therapeutic response. The current research discusses the contribution of CSLM in the diagnosis and assessment of diabetes, neurodegenerative diseases, rheumatology, immunology, and chemotherapy. Indeed, further work is needed to evaluate its potential use in the diagnosis and management of the systemic disease. 

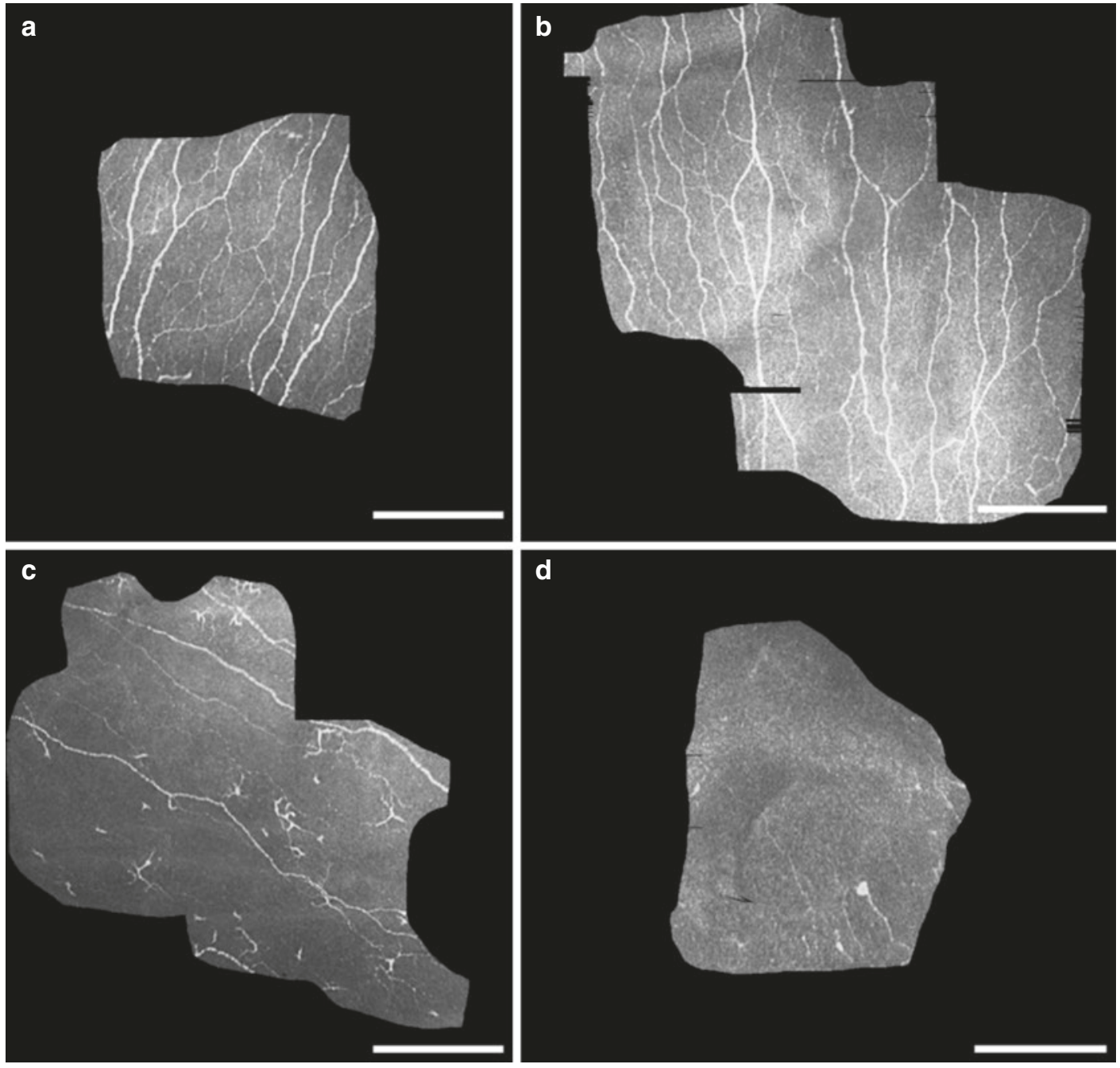

Fig. 12.11 Confocal images demonstrating the morphology of SNP from two control subjects $(\mathbf{a}, \mathbf{b})$ and two Charcot subjects $(\mathbf{c}, \mathbf{d})$. $\mathbf{c}$ and $\mathbf{d}$ exhibited readily visible changes with decrease in nerve fibers, decrease in nerve branches and connectivity, and presence of widely scat- tered dendritic cells (c). Each scale bar represents $200 \mu \mathrm{m}$. (In cooperation with Herlyn \& Mittlmeier (University of Rostock) as well as Köhler \& Allgeier (Karlsruhe Institute of Technology))

\subsection{Non-ophthalmological Applications}

The application of cSLM in non-transparent tissue is limited due to light interaction with human tissue. Light-tissue interactions include reflection and refraction (when light encounters dif- ferent types of tissue), absorption and scattering of photons. The absorption of photons depends on factors like electron structure of atoms and molecules in the material, the wavelength of the light and the temperature. In biological tissue it is mainly caused by water molecules and macromolecules such as proteins and chromophores. 

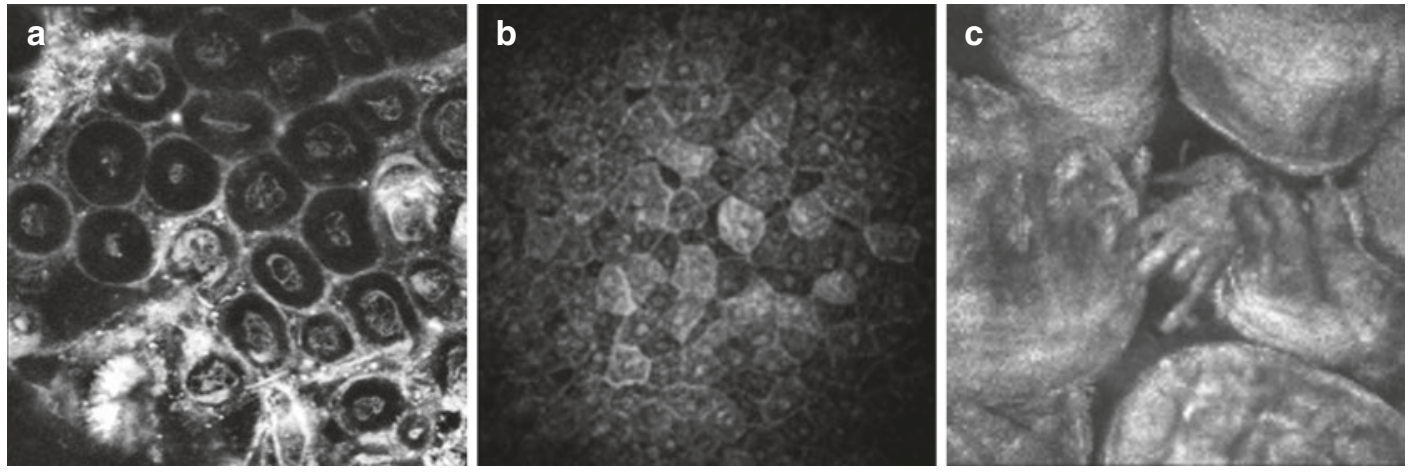

Fig. 12.12 cSLM in Gynaecology (a, vulva dysplasia, in vitro), in dermatology (b, skin epithelium, in vivo) and in Otorhinolaryngology (c, taste buds, in vivo)

Tissues are very heterogeneous materials that have spatial fluctuations in their optical properties due to variations in density and refractive index. As a consequence they strongly scatter light and are non-transparent. Imaging of such turbid media is limited by the penetration depth of light. Therefore, cSLM images with cellular resolution can only be obtained at depths up to $300 \mu \mathrm{m}$. In Fig. 12.12 tissue imaging in gynecology, dermatology, and otorhinolaryngology is demonstrated.

Several studies have been performed to assess the ability to image the characteristics of special tissue properties. Very interestingly, cSLM has been used to describe the cellular morphology and pathological alterations of the oral cavity, cervix, and esophagus [55-57]. Reflectance imaging of human skin can provide insights of cell morphology and tissue architecture of the epithelium [58, 59].

Furthermore, a number of pathological skin conditions were investigated [60, 61]. CSLM was also performed in the amelanotic epithelial tissue of the gastrointestinal tract [57], lip and tongue [55] and the oropharynx [62]. CSLM can be used as a non-invasive tool in the diagnosis

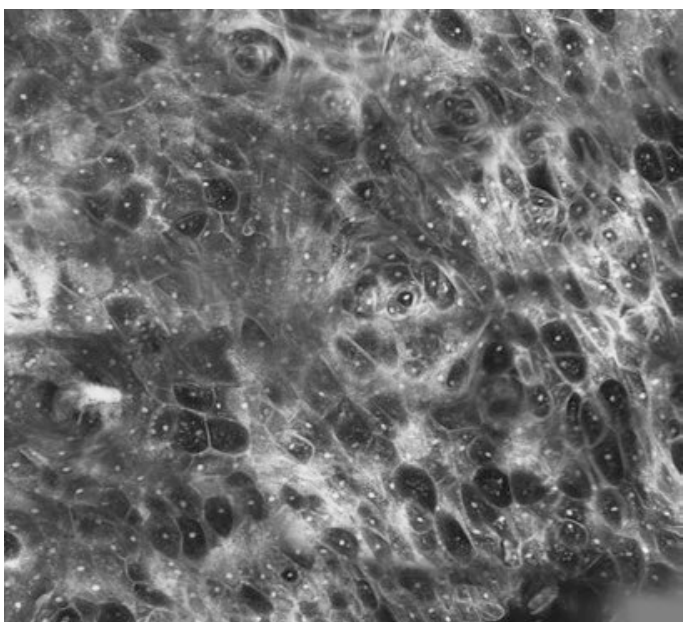

Fig. 12.13 Mosaicking cSLM in Otorhinolaryngology showing cellular structure of the mucous membrane (in vivo, $2.5 \mathrm{~mm} \times 2.5 \mathrm{~mm}$ )

of sinonasal inverted papilloma, see Figs. 12.13 and 12.14 [63].

A promising application is endoscopic cSLM imaging for diagnostics in schistosomiasis. We were able to detect schistosomal eggs in the urothelium of a patient with urinary schistosomiasis as is demonstrated in Fig. 12.15 [64, 65]. 


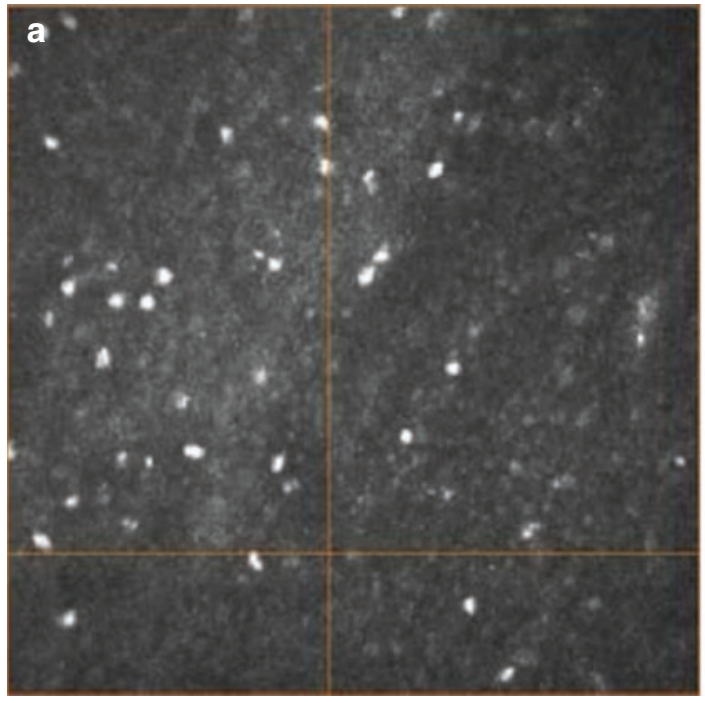

Fig. 12.14 Upper (a) and deeper (b) part of the cylindric epithelium of nasal polyps. The almost nucleus free cell bodies show a homogenous appearance (a), whereas the

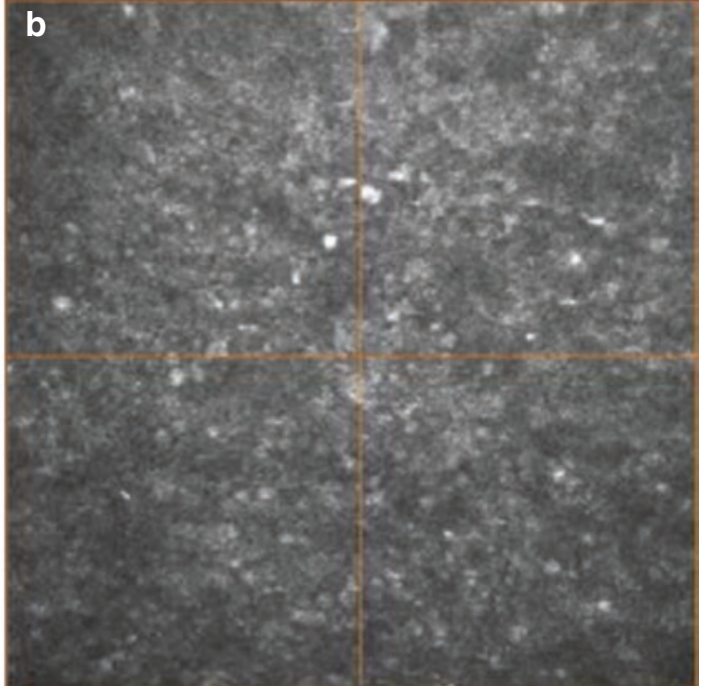

nuclei are densely packed and homogenously organized near the basal membrane (b)
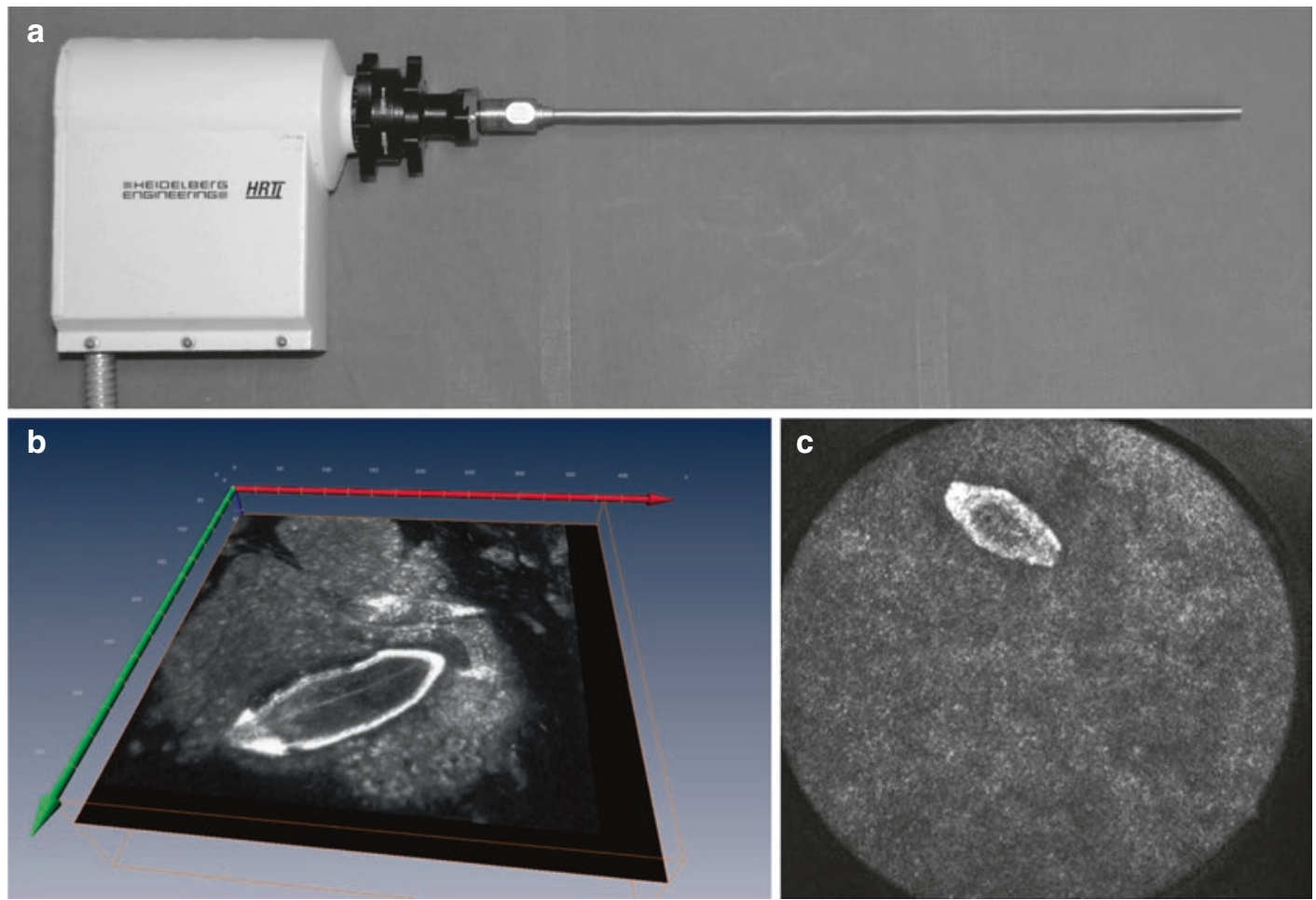

Fig. 12.15 Endoscopic image modality based on the Heidelberg Retina Tomograph (HRTII) (a), eggs of Schistosoma mansoni visualized within the mucosal tissue of the large intestine (b) and cSLM of the bladder showing eggs of Schistosoma haematobium (c) (in cooperation with Holtfretter, Fritsche und Reisinger, University of Rostock) 


\subsection{Current and Future Developments}

Nowadays, in vivo corneal confocal microscopy has received a high level of scientific and clinical attention in ophthalmology. The ability to acquire high-resolution images of various cellular structures inside the living cornea non-invasively has inspired the idea of using this technology for diagnostic purposes. A number of innovations are under development in order to assess their diagnostic potential and the usability of the technology.

\subsubsection{Subbasal Nerve Plexus Mosaicking}

A special focus has been given on the assessment of corneal nerves and their involvement in ocular and systemic diseases, especially for noninvasive and repeatable techniques which can quantify ocular neurodegenerative changes in individuals with diabetes [12].

Results of older studies in this context were always based on morphometric parameter values derived from single cSLM image with a field of view in the order of $400 \times 400 \mu \mathrm{m}^{2}$ each. Recent examinations suggest that a robust morphometric assessment requires the analysis of larger areas of the central cornea in order to compensate for a locally inhomogeneous arrangement of the SNP [66, 67].

In this context, a concept for automated and fast control of the focal plane was developed which allows for mosaicking of the subbasal nerve plexus and for increasing the reliability of quantification [68]. This new approach uses a modified RCM in combination with software routines for real-time image acquisition. Furthermore, a new optical design was developed using a piezo actuator which moves a lens inside the modified RCM to control the focal plane without moving the TomoCap. During image acquisition, the focus plane oscillates around the SNP between the basal epithelium and the anterior stroma and uses a cornea tissue classification algorithm

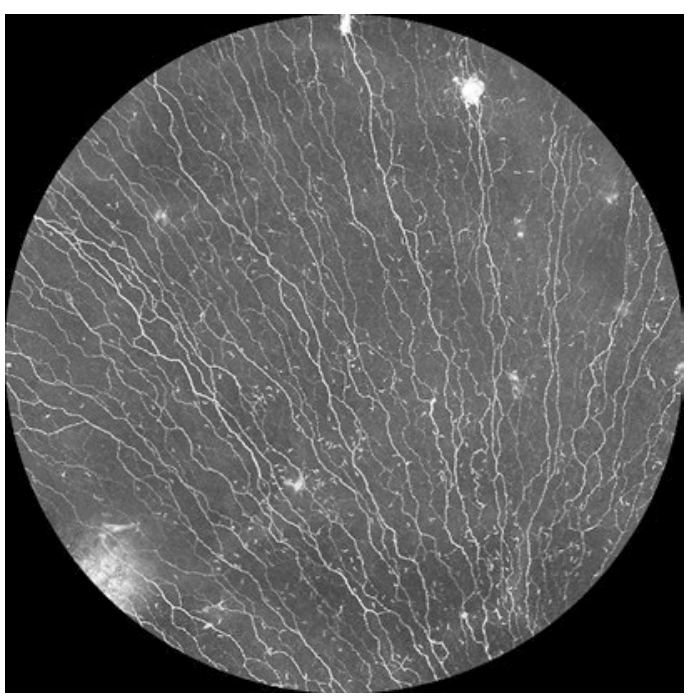

Fig. 12.16 Large scale SNP mosaicking of a healthy human subject

(CTC). This algorithm distinguishes different tissues and thus delivers the optimal offset position for the piezo oscillation around the SNP. With the new piezo-based RCM fast and defined focal plane shifts are possible. As investigations have shown, the optimized CTC is fast enough for real-time usage. Preliminary results also suggest that the CTC can significantly increase the quality of SNP mosaics through the exclusion of images from other cell layers. The presented concept is promising for large-scale SNP mosaicking (see Fig. 12.16). This will mark a necessary step towards reliable SNP quantification, a promising biomarker for diabetic peripheral neuropathy.

\subsubsection{Slit Lamp Microscopy on a Cellular Level Using In Vivo Confocal Laser Scanning Microscopy}

Recently, we have presented an in vivo method for volumetric reconstruction of the cornea on a cellular level with volume sizes up to around $250 \times 300 \times 400 \mu \mathrm{m}^{3}$ [69]. For image acquisition the microscope objective is equipped with a piezo 
actuator. The automated, closed-loop control of the focal plane enables fast and precise focus positioning. Additionally, a novel contact cap with a concave surface is presented. It clearly reduces eye movements, thereby increasing the cuboid volume of the generated $3 \mathrm{D}$ reconstruction significantly (see Fig. 12.17). Using the isotropic volume stacks sectional views of any orientation can be generated which opens the window to slit lamp microscopy on a cellular level (see Fig. 12.18).

\subsubsection{OCT-Guided In Vivo Confocal Laser Scanning Microscopy}

Corneal confocal microscopy became a valuable tool for studying the corneal morphology and offers non-invasive in vivo imaging at the cellular level being important for current research. However, the technique is not only limited by the small field of view. It is also difficult to specify the exact cSLM image
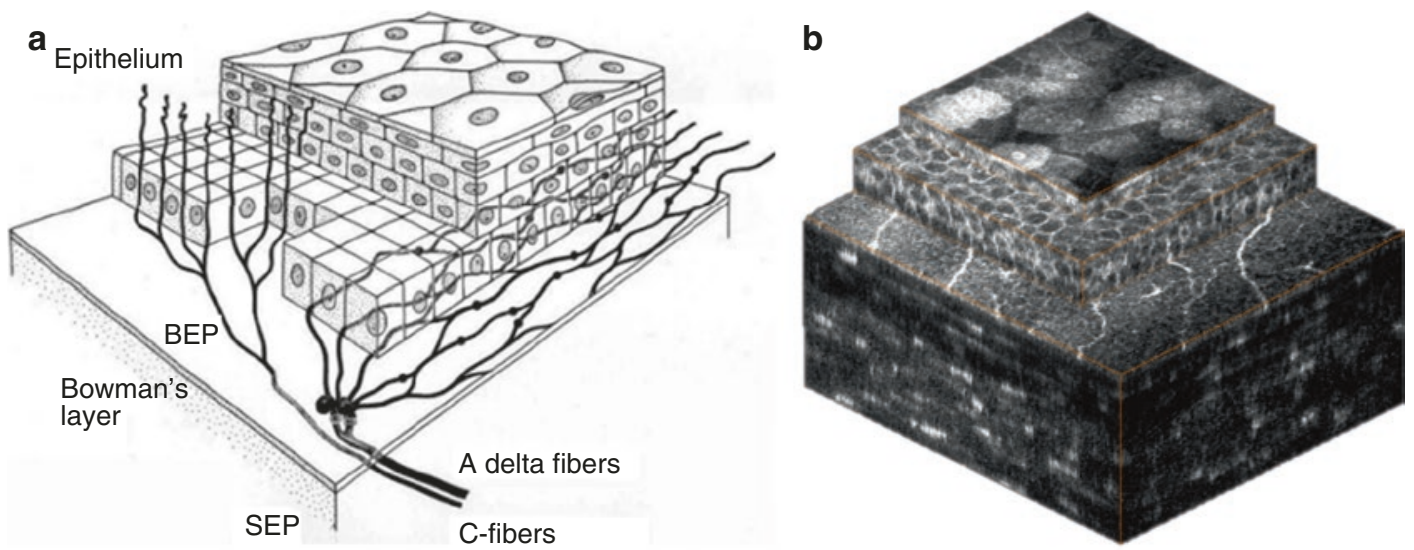

Fig. 12.17 The anterior part of the cornea: sketch (a) according to Guthoff et al. [70] and 3D reconstruction (b) according to Bohn et al. [69]
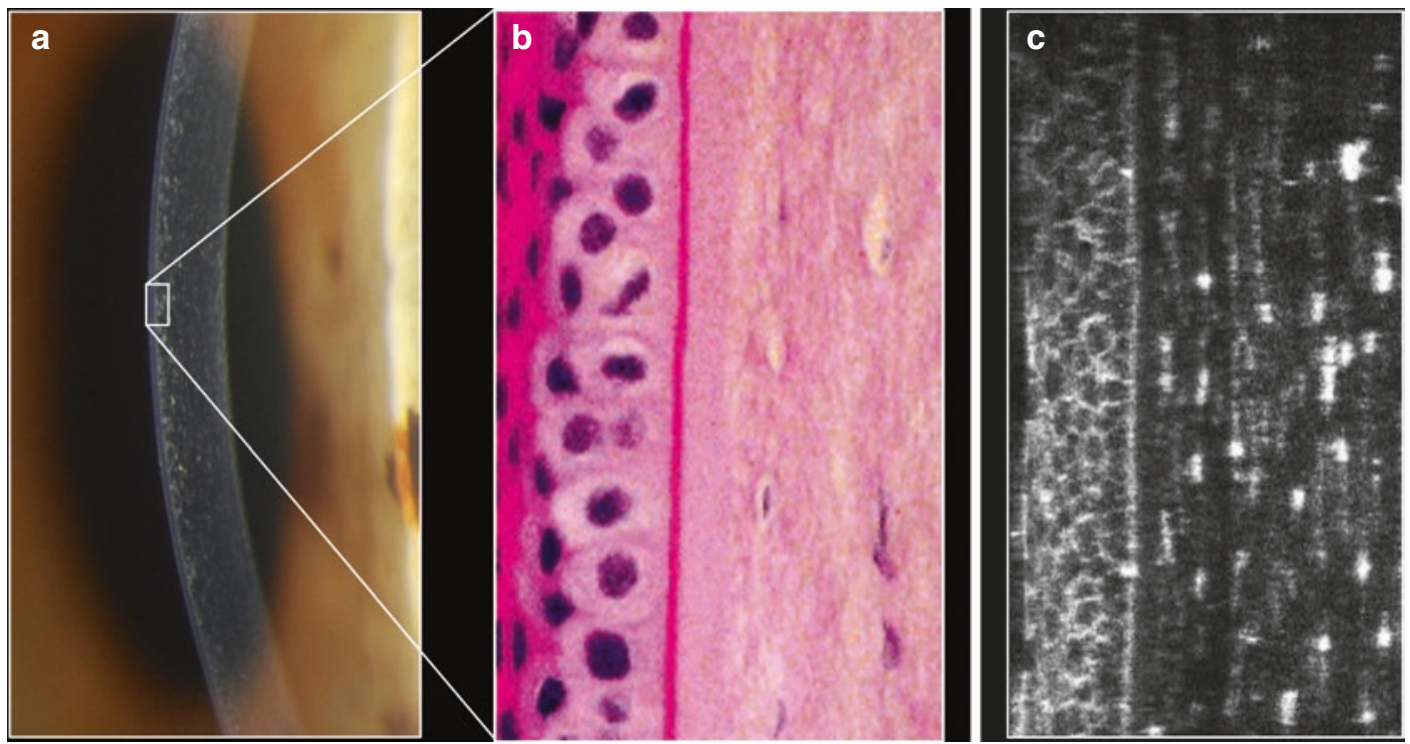

Fig. 12.18 Conventional slit lamp (a), histology (b) and cross-section after 3D-reconstruction (c) exemplifying the potential of the laser based slit lamp 
location and orientation inside the cornea. To overcome this limitation, a commercially available multimodal imaging platform was adapted for in vivo OCT-guided cSLM. A microscope lens was attached to a SPECTRALIS with OCT2 module (Heidelberg Engineering, Germany) using a customized, modular lens adapter and a piezo actuator for computerized focus control. The light sources of both modalities (cSLM and OCT) are combined within the camera head, and have a common beam path through the SPECTRALIS objective and the added microscope lens. The optical path length of the OCT reference beam was changed to account for the additional optical component. Multimodal imaging could be performed simultaneously with $8.9 \mathrm{fps}$ and a field of view of $805 \times 805 \mu \mathrm{m}^{2}$ for cSLM (xy-image) and with $90 \mathrm{fps}$ and a field of view of $805 \times 1919 \mu \mathrm{m}^{2}$ for OCT (B-Scan, xz-section). Due to the high numerical aperture of the microscope lens the depth of field is very limited for both images, the cSLM and the OCT. The focal plane can be recognized in the OCT cross-section as bright surface, which reveals the actual depth position of the cSLM image (see Fig. 12.19). Simultaneously, the cornea's anterior and posterior interface can be visualized because of their strong backscattering and the high sensitivity of OCT.
The piezo actuator can shift the cSLM focal plane up to $600 \mu \mathrm{m}$ whereas the image position and orientation can be tracked in real-time. Compared to the conventional state of the art, the OCT-guided cSLM concept significantly improved the usability. Real-time assessment of the cSLM image plane location and orientation inside the cornea by means of the OCT cross-section enables an improved location-based diagnosis. For the first time, it is possible to specify the angle between the corneal surface and the cSLM image. Further effort is necessary for optimizing the system design and OCT scan patterns.

\subsubsection{Multiphoton Microscopy}

Corneal cell differentiation in vivo can be performed only on a morphological basis, and in the majority of cases, this is not sufficient using cSLM. This window could be opened by multiphoton microscopy. The non-linear interaction mechanisms, such as multiphoton absorption or frequency conversion, can be induced by using a highly focused pulsed laser. Using this advanced technology, induced autofluorescence, second-harmonic generation or fluorescence lifetime measurements can be used to produce cellspecific information with subcellular resolution [71-73].

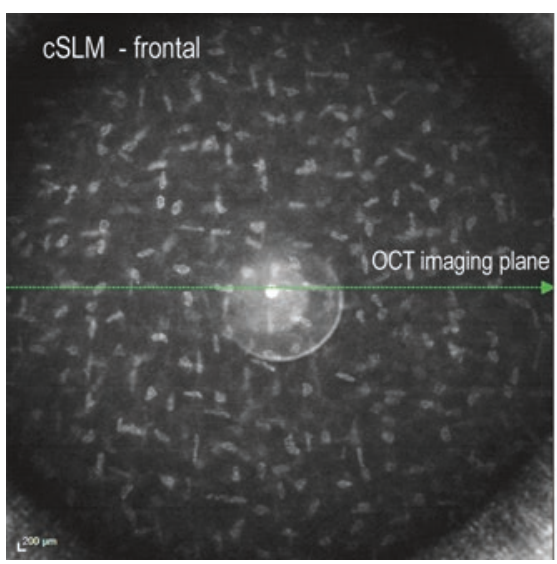

Fig. 12.19 Example for OCT guided cSLM: confocal image (left) and cross-sectional OCT image (right). The bright reflection within the OCT image reveals the posi- tion of the confocal en-face image with respect to the cornea's anterior and posterior interface 


\subsection{Summary}

In summary, state-of-the-art cSLM allows to evaluate the ocular surface at a cellular level with subsequent 2D mosaicking or 3D reconstruction. But only a close and straightforward cooperation between basic as well as clinical science and industry partners may bring about the fulfillment of the great promise offered by this technology.

\section{References}

1. Stave J, et al. Modified Heidelberg Retinal Tomograph HRT. Initial results of in vivo presentation of corneal structures. Ophthalmologe. 2002;99(4):276-80.

2. Labbé A, et al. Comparative anatomy of laboratory animal corneas with a new-generation highresolution in vivo confocal microscope. Curr Eye Res. 2006;31(6):501-9.

3. Mazzotta C, et al. Treatment of progressive keratoconus by riboflavin-UVA-induced cross-linking of corneal collagen: ultrastructural analysis by Heidelberg Retinal Tomograph II in vivo confocal microscopy in humans. Cornea. 2007;26(4):390-7.

4. Petroll WM, et al. Quantitative 3-D corneal imaging in vivo using a modified HRT-RCM confocal microscope. Cornea. 2013;32(4):e36.

5. Allgeier $\mathrm{S}$, et al. Image reconstruction of the subbasal nerve plexus with in vivo confocal microscopy. Invest Ophthalmol Vis Sci. 2011;52(9):5022-8.

6. Zhivov A, et al. Real-time mapping of the subepithelial nerve plexus by in vivo confocal laser scanning microscopy. Br J Ophthalmol. 2010;94(9): 1133-5.

7. Turuwhenua JT, Patel DV, McGhee CN. Fully automated montaging of laser scanning in vivo confocal microscopy images of the human corneal subbasal nerve plexus. Invest Ophthalmol Vis Sci. 2012;53(4):2235-42.

8. Edwards K, et al. Wide-field assessment of the human corneal subbasal nerve plexus in diabetic neuropathy using a novel mapping technique. Cornea. 2012;31(9):1078-82.

9. Poletti E, et al. Automatic montaging of corneal sub-basal nerve images for the composition of a wide-range mosaic. In: Engineering in Medicine and Biology Society (EMBC), 2014 36th Annual International Conference of the IEEE. IEEE; 2014.

10. Allgeier S, et al. Mosaicking the subbasal nerve plexus by guided eye movements. Invest Ophthalmol Vis Sci. 2014;55(9):6082-9.

11. Köhler B, et al. Large-scale imaging of corneal nerve fibres by guided eye movements. Klin Monbl Augenheilkd. 2014;231(12):1170-3.
12. De Clerck EE, et al. New ophthalmologic imaging techniques for detection and monitoring of neurodegenerative changes in diabetes: a systematic review. Lancet Diabet Endocrinol. 2015;3(8):653-63.

13. Li HF, et al. Epithelial and corneal thickness measurements by in vivo confocal microscopy through focusing (CMTF). Curr Eye Res. 1997;16(3):214-21.

14. Stachs O, et al. In vivo three-dimensional confocal laser scanning microscopy of the epithelial nerve structure in the human cornea. Graefes Arch Clin Exp Ophthalmol. 2007;245(4):569-75.

15. Zhivov A, et al. In vivo three-dimensional confocal laser scanning microscopy of corneal surface and epithelium. Br J Ophthalmol. 2009;93(5):667-72.

16. Minsky M. Memoir on inventing the confocal scanning microscope. Scanning. 1988;10(4):128-38.

17. Webb RH, Hughes GW, Delori FC. Confocal scanning laser ophthalmoscope. Appl Opt. 1987;26(8):1492-9.

18. Cavanagh HD, et al. Confocal microscopy of the living eye. CLAO J. 1990;16(1):65-73.

19. Petráň M, et al. Tandem-scanning reflected-light microscope. J Opt Soc Am. 1968;58(5):661-4.

20. Wiegand W, et al. Optical sectioning of the cornea with a new confocal in vivo slit-scanning videomicroscope. Ophthalmology. 1995;102(4):568-75.

21. Masters BR, Thaer AA. Real-time scanning slit confocal microscopy of the in vivo human cornea. Appl Opt. 1994;33(4):695-701.

22. Wilson T, Sheppard C. Theory and practice of scanning optical microscopy, vol. 180. London: Academic; 1984.

23. Conchello J-A, Lichtman JW. Optical sectioning microscopy. Nat Methods. 2005;2(12):920.

24. Jalbert I, et al. In vivo confocal microscopy of the human cornea. Br J Ophthalmol. 2003;87(2):225-36.

25. Tuchin VV. Tissue optics: light scattering methods and instruments for medical diagnosis. Washington, DA: SPIE-International Society for Optical Engineering; 2007.

26. Wilson B, Adam G. A Monte Carlo model for the absorption and flux distributions of light in tissue. Med Phys. 1983;10(6):824-30.

27. Meek KM, Knupp C. Corneal structure and transparency. Prog Retin Eye Res. 2015;49:1-16.

28. Guthoff RF, Baudouin C, Stave J. Atlas of confocal laser scanning in-vivo microscopy in ophthalmology. Berlin: Springer Science \& Business Media; 2007.

29. Houang E, et al. Microbial keratitis in Hong Kong: relationship to climate, environment and contactlens disinfection. Trans R Soc Trop Med Hyg. 2001;95(4):361-7.

30. Zhivov A, et al. Diabetic foot syndrome and corneal subbasal nerve plexus changes in congolese patients with type 2 diabetes. PLoS One. 2015;10(3):e0119842.

31. Ziegler D, et al. Early detection of nerve fiber loss by corneal confocal microscopy and skin biopsy in recently diagnosed type 2 diabetes. Diabetes. 2014;63(7):2454-63.

32. Niederer RL, et al. Age-related differences in the normal human cornea: a laser scanning in vivo 
confocal microscopy study. $\mathrm{Br}$ J Ophthalmol. 2007;91(9):1165-9.

33. Hahnel C, et al. The keratocyte network of human cornea: a three-dimensional study using confocal laser scanning fluorescence microscopy. Cornea. 2000;19(2):185-93.

34. Hovakimyan M, et al. Morphological analysis of quiescent and activated keratocytes: a review of ex vivo and in vivo findings. Curr Eye Res. 2014;39(12):1129-44.

35. Petroll W, Cavanagh H, Jester J. Three-dimensional imaging of corneal cells using in vivo confocal microscopy. J Microsc. 1993;170(3):213-9.

36. Jester JV, et al. Application of in vivo confocal microscopy to the understanding of surfactantinduced ocular irritation $*$ Oryzias latipes. Toxicol Pathol. 1996;24(4):412-28.

37. Grupcheva $\mathrm{CN}$, et al. In vivo and ex vivo in situ confocal analysis of a rat model demonstrating transient 'epithelialization of the endothelium'. Clin Exp Ophthalmol. 2002;30(3):191-5.

38. Song J, et al. Neonatal corneal stromal development in the normal and lumican-deficient mouse. Invest Ophthalmol Vis Sci. 2003;44(2):548-57.

39. Jester JV, et al. Measurement of corneal sublayer thickness and transparency in transgenic mice with altered corneal clarity using in vivo confocal microscopy. Vis Res. 2001;41(10-11):1283-90.

40. Reichard M, et al. Comparative in vivo confocal microscopical study of the cornea anatomy of different laboratory animals. Curr Eye Res. 2010;35(12): 1072-80.

41. Maurer JK, et al. Confocal microscopic characterization of initial corneal changes of surfactant-induced eye irritation in the rabbit. Toxicol Appl Pharmacol. 1997;143(2):291-300.

42. Ivarsen A, Laurberg T, Møller-Pedersen T. Role of keratocyte loss on corneal wound repair after LASIK. Invest Ophthalmol Vis Sci. 2004;45(10):3499-506.

43. Ivarsen A, Møller-Pedersen T. LASIK induces minimal regrowth and no haze development in rabbit corneas. Curr Eye Res. 2005;30(5):363-73.

44. Chang JH, et al. The application of in vivo confocal microscopy and tear LDH measurement in assessing corneal response to contact lens and contact lens solutions. Curr Eye Res. 1999;19(2):171-81.

45. Ichijima $\mathrm{H}$, et al. Effects of increasing $\mathrm{Dk}$ with rigid contact lens extended wear on rabbit corneal epithelium using confocal microscopy. Cornea. 1992;11(4):282-7.

46. Leckelt J, et al. Early detection of diabetic neuropathy by investigating CNFL and IENFD in thy 1-YFP mice. J Endocrinol. 2016;231(2):147-57.

47. Leppin K, et al. Diabetes mellitus leads to accumulation of dendritic cells and nerve fiber damage of the subbasal nerve plexus in the cornea. Invest Ophthalmol Vis Sci. 2014;55(6):3603-15.

48. Reddy VC, et al. Corneal sensitivity, blink rate, and corneal nerve density in progressive supranuclear palsy and Parkinson disease. Cornea. 2013;32(5):631-5.
49. Kinard KI, et al. Chronic migraine is associated with reduced corneal nerve fiber density and symptoms of dry eye. Headache. 2015;55(4):543-9.

50. Bitirgen G, et al. Use of corneal confocal microscopy to detect corneal nerve loss and increased dendritic cells in patients with multiple sclerosis. JAMA Ophthalmol. 2017;135(7):777-82.

51. Petropoulos IN, et al. Corneal confocal microscopy: an imaging endpoint for axonal degeneration in multiple sclerosis. Invest Ophthalmol Vis Sci. 2017;58(9):3677-81.

52. Rousseau A, et al. Potential role of in vivo confocal microscopy for imaging corneal nerves in transthyretin familial amyloid polyneuropathy. JAMA Ophthalmol. 2016;134(9):983-9.

53. Tavakoli M, et al. Corneal confocal microscopy: a novel noninvasive test to diagnose and stratify the severity of human diabetic neuropathy. Diabetes Care. 2010;33(8):1792-7.

54. Lovblom LE, et al. In vivo corneal confocal microscopy and prediction of future-incident neuropathy in type 1 diabetes: a preliminary longitudinal analysis. Can J Diabetes. 2015;39(5):390-7.

55. White WM, et al. Noninvasive imaging of human oral mucosa in vivo by confocal reflectance microscopy. Laryngoscope. 1999;109(10):1709-17.

56. Drezek RA, et al. Laser scanning confocal microscopy of cervical tissue before and after application of acetic acid. Am J Obstet Gynecol. 2000;182(5):1135-9.

57. Inoue $\mathrm{H}$, et al. A novel method of virtual histopathology using laser-scanning confocal microscopy in-vitro with untreated fresh specimens from the gastrointestinal mucosa. Endoscopy. 2000;32(06):439-43.

58. Bertrand C, Corcuff P. In vivo spatio-temporal visualization of the human skin by real-time confocal microscopy. Scanning. 1994;16(3):150-4.

59. Rajadhyaksha M, Anderson RR, Webb RH. Videorate confocal scanning laser microscope for imaging human tissues in vivo. Appl Opt. 1999;38(10):2105-15.

60. González S, et al. Characterization of psoriasis in vivo by reflectance confocal microscopy. J Med. 1999;30(5-6):337-56.

61. Busam KJ, et al. Morphologic features of melanocytes, pigmented keratinocytes, and melanophages by in vivo confocal scanning laser microscopy. Mod Pathol. 2001;14(9):862.

62. Just $\mathrm{T}$, et al. Laser scanning microscopy of the human larynx mucosa: a preliminary, ex vivo study. Laryngoscope. 2006;116(7):1136-41.

63. Óvári A, et al. Optical coherence tomography and confocal laser scanning microscopy as non-invasive tools in the diagnosis of sinonasal inverted papilloma: a pilot study. Eur Arch Otorhinolaryngol. 2018;275(7):1775-81.

64. Holtfreter MC, et al. Confocal laser scanning microscopy for detection of Schistosoma mansoni eggs in the gut of mice. PLoS One. 2011;6(4):e18799. 
65. Fritzsche $\mathrm{C}$, et al. Confocal laser scanning microscopy, a new in vivo diagnostic tool for schistosomiasis. PLoS One. 2012;7(4):e34869.

66. Winter $\mathrm{K}$, et al. Local variability of parameters for characterization of the corneal subbasal nerve plexus. Curr Eye Res. 2016;41(2):186-98.

67. Allgeier S, et al. A novel approach to analyze the progression of measured corneal sub-basal nerve fiber length in continuously expanding mosaic images. Curr Eye Res. 2017;42(4):549-56.

68. Bohn S, et al. Concepts for automated fast focal plane control in subbasal nerve plexus mosaicking to reliably quantify a biomarker for diabetic peripheral neuropathy. Invest Ophthalmol Vis Sci. 2017;58(8):1431.

69. Bohn S, et al. Cellular in vivo 3D imaging of the cornea by confocal laser scanning microscopy. Biomed Opt Express. 2018;9(6):2511-25.
70. Guthoff RF, et al. Epithelial innervation of human cornea: a three-dimensional study using confocal laser scanning fluorescence microscopy. Cornea. 2005;24(5):608-13.

71. Krüger A, et al. Combined nonlinear and femtosecond confocal laser-scanning microscopy of rabbit corneas after photochemical cross-linking. Invest Ophthalmol Vis Sci. 2011;52(7):4247-55.

72. Ehmke T, et al. Nonlinear microscopy in ophthalmology: principles and pathbreaking applications. Klin Monsbl Augenheilkd. 2015;232(12):1365-73.

73. Ehmke T, et al. In vivo nonlinear imaging of corneal structures with special focus on BALB/c and streptozotocin-diabetic Thy1-YFP mice. Exp Eye Res. 2016;146:137-44.

Open Access This chapter is licensed under the terms of the Creative Commons Attribution 4.0 International License (http://creativecommons.org/licenses/by/4.0/), which permits use, sharing, adaptation, distribution and reproduction in any medium or format, as long as you give appropriate credit to the original author(s) and the source, provide a link to the Creative Commons license and indicate if changes were made.

The images or other third party material in this chapter are included in the chapter's Creative Commons license, unless indicated otherwise in a credit line to the material. If material is not included in the chapter's Creative Commons license and your intended use is not permitted by statutory regulation or exceeds the permitted use, you will need to obtain permission directly from the copyright holder. 